\title{
THE LINEAR ELLIPTIC - SURFACES OF REVOLUTION
}

The paper presents a category of revolution surfaces which may be generated by the metrical Euclidean transformation compounded by revolutions and displacements. These surfaces are created by a straight line revolution around a revolution axis moving on an ellipse. This surface group is classified according to the relative positions of a revolution axis moving in contact with an ellipse, and according to the relative positions of a straight line and a revolution axis. The straight line revolution and axis motion can be for equal or different orientation and both can have equal or different radian frequencies. The transformation matrices for the vector equations of the surface and also some properties of these surfaces are presented in this paper.

\section{Introduction}

The linear elliptic-surface of revolution is generated by a straight line revolution around the revolution axis $o$ moving on the ellipse $k$ and it has the specified direction with respect to the ellipse and one point $R=a \cap k$ lies on the ellipse $k$. We can create the corresponding metrical transformation for this revolution by the composition of three-dimensional transformations, a revolution and displacement. We can represent this transformation by a regular square matrix of $4^{\text {th }}$ dimension whose elements are the real functions of one variable

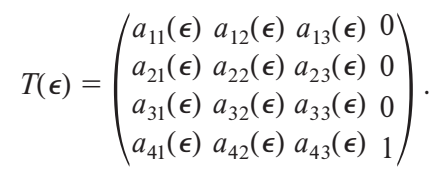

We may express the vector equation of this revolution surface as a product of the vector function of the straight line $p r(t)=$ $(x(t) y(t) z(t) 1), t \in\langle-\infty,+\infty\rangle$ and the corresponding transformation matrix $T(\epsilon): p(\epsilon, t)=r(t) . T(\epsilon)$.

\section{Classification of surfaces}

The above described linear revolution surfaces may be classified according to the relative positions of the revolution axis $o$ and the ellipse $k=(0, a, b) \subset x z$ with parametric equations

$$
\begin{aligned}
& x=a \cos \epsilon \\
& y=0 \\
& z=b \sin \epsilon
\end{aligned}, \epsilon \in\langle 0,2 \pi\rangle
$$

to the five subgroups:
I. $o \| z$
$(z$ - minor axis of ellipse $k)$
II. $o \| x$
( $x$ - major axis of ellipse $k$ ),
III. $o=n$
( $n$ - a normal to the ellipse $k$ ),
IV. $o=t$
( $t$ - tangent to the ellipse $k$ ),

$$
\text { V. o } \| \mathrm{y} \quad(y \text { - perpendicular to the plane } x z) \text {. }
$$

In case of I - IV subgroups, the revolution axis o lies in the plane of ellipse $k \subset x z \perp y$ and these surfaces are of the spherical (when $o \times y$ ) or Euler type (when $0 / y$ ), in case of V subgroup the axis of revolution $o$ is perpendicular to the plane of the ellipse $o \perp x z$ and these surfaces are of the cycloidal type.

Next, these surfaces may be classified according to the relative position of the straight line $\mathrm{p}$ and the revolution axis $\mathrm{o}$ :
a) cylindrical
$p \| o$
(parallel),
b) conical
$p \times o$
(concurrent),
c) hyperbolical
$p / o$
(skew).

\subsection{Surfaces of the subgroup I ( $o$ is parallel to the minor axis of the ellipse $k$ )}

We will formulate the transformation matrix of the revolution surface, which is created by a revolution around the axis $o \| z$ as a product of matrices, which represents the displacement parallel to the vector $(-a, 0,0)$, the revolution through the angle $\epsilon^{\prime}= \pm \mathrm{m} \epsilon$, $\epsilon \in\langle 0,2 \pi\rangle$ about the coordinate axis $z$ and displacement parallel to the vector ( $a \cos \epsilon, 0, b \sin \epsilon$ ), where the addition sign ( + ) is in the case of equal orientation, the minus sign $(-)$ is in the case of different orientations of the line $p$ revolution and the axis $o$ motion, the coefficient $m$ is a multiple of the radian frequency of the axis $o$ motion of

$$
T(\epsilon)=\left(\begin{array}{cccc}
\cos \epsilon^{\prime} & \sin \epsilon^{\prime} & 0 & 0 \\
-\sin \epsilon^{\prime} & \cos \epsilon^{\prime} & 0 & 0 \\
0 & 0 & 1 & 0 \\
a\left(\cos \epsilon-\cos \epsilon^{\prime}\right) & -a \sin \epsilon^{\prime} & b \sin \epsilon & 1
\end{array}\right) .
$$

If the straight line $p$ parallel to the axis $o$ (Fig. 1) is determined by the point $A\left(x_{A}, 0,0\right)$, where $x_{A} \neq a$, then its vector equation is $r(t)=\left(x_{A}, 0, t, 1\right)$. In Fig. 2 the surface of the cylindrical type for a sign ( - ) of orientation of motions and for $m=6$ is shown.

\footnotetext{
* Tatiana Olejníková

Department of Mathematics and Descriptive Geometry, Faculty of Civil Engineering, Technical University, Vysokoškolská 4, 04001 Košice,

E-mail: tatiana.olejnikova@tuke.sk
} 


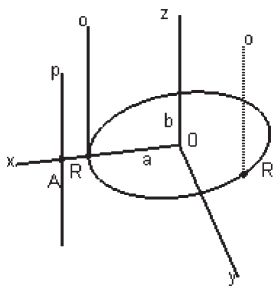

Fig. 1

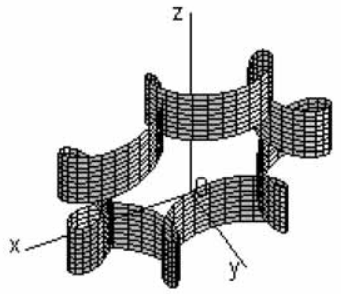

Fig. 2

If the straight line $p$ concurrent to the axis $o$ is determined by the point $A\left(a, y_{4}, z_{A}\right)$, and the distance $v$ of the point $C=p \cap x z$ from the axis $x, v=\left|C_{x}\right|$, we will express it by the vector equation $r(t)=\left(a, y_{A}(1-t), t v, 1\right)$ (Fig. 3).

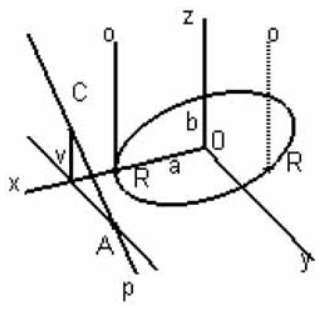

Fig. 3

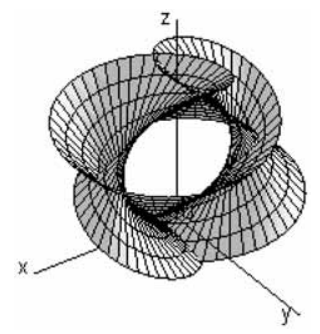

Fig. 4

If $p \perp o, p \perp x$, then the surface includes an ellipse $k$ and two congruent ellipses located in mutually perpendicular planes (Fig. $5)$. For $p \perp o, p=x$, the surface is elliptic conoid of $4^{\text {th }}$ degree, where the generating line is perpendicular to the plane of the generating ellipse (Fig. 6).

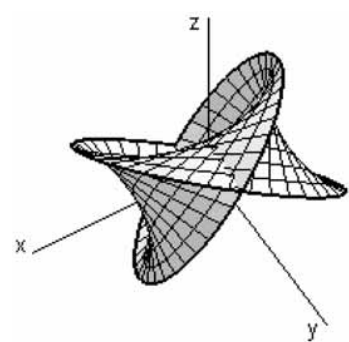

Fig. 5

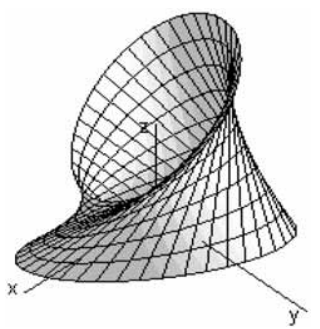

Fig. 7

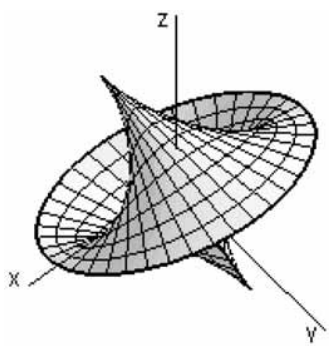

Fig. 6

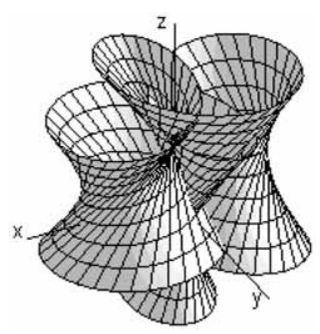

Fig. 8
The straight line $p$ skew to the axis $o$ (Fig. 3) is given by the point $A\left(x_{A}, y_{A}, z_{A}\right), x_{A} \neq a$ and by distance of the point $C=p \cap x z$ from the axis $x, v=|C x| \neq 0$. We can express the straight line $p$ by the vector equation $r(t)=\left(x_{A}, y_{A}(1-t), z_{A}+t\left(v-z_{A}\right), 1\right)$. Surfaces of the hyperbolic type for $m=1$ and $m=3$ are shown in Figs. 7, 8 .

\subsection{Surfaces of the subgroup II ( 0 is parallel to the major axis of the ellipse $k$ )}

We will formulate the transformation matrix of the revolution surface where $o \| x$ as a product of matrices, which represent the displacement parallel to the vector $(-a, 0,0)$, the revolution through the angle $\epsilon^{\prime}= \pm m \epsilon, \epsilon \in\langle 0,2 \pi\rangle$ about the coordinate axis $x$ and displacement parallel to the vector $(a \cos \epsilon, 0, b \sin \epsilon)$

$$
T(\epsilon)=\left(\begin{array}{cccc}
1 & 0 & 0 & 0 \\
0 & \cos \epsilon^{\prime} & -\sin \epsilon^{\prime} & 0 \\
0 & \sin \epsilon^{\prime} & \cos \epsilon^{\prime} & 0 \\
a(\cos \epsilon-1) & 0 & b \sin \epsilon & 1
\end{array}\right) .
$$

If the straight line $p$ is parallel to the axis o (Fig. 9) and given by the point $A\left(x_{A}, y_{A}, z A\right), y_{A} \neq 0$, its vector equation is $r(t)=$ $=\left(x_{A}(1-t), y_{A}, z_{A}, 1\right)$. In Fig. 10 the surface of the cylindrical type for a sign $(-)$ of orientation of motions and is shown.

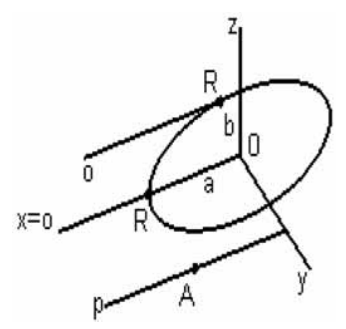

Fig. 9

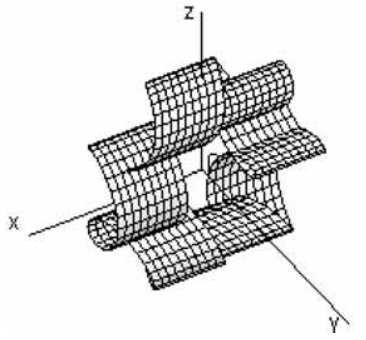

Fig. 10
If the straight line $p$ is concurrent to the axis $o$ (Fig. 11), given by the points $A\left(x_{A}, 0, z_{A}\right)$ and $C(a, 0,0)$, then it creates the surface of the conical type, which is in Fig. 12 for $m=5$ and for sign (-) for the orientation of movements. Because the point $C=a \cap k$, then the surface contains the ellipse $k$.

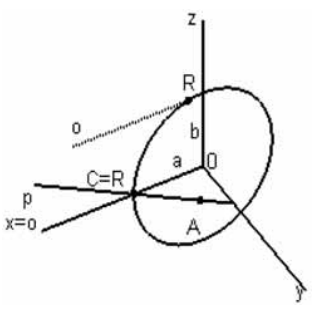

Fig. 11

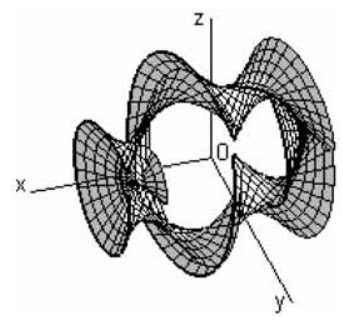

Fig. 12
If the straight line $p$ is skew to the axis $o$ (Fig. 13), given by the points $A\left(x_{A}, y_{A}, z_{A}\right)$ and $C\left(a, y_{A}, 0\right)$, then it creates the surface 
of the hyperbolical type, which in Fig. 14 for $m=3$ and for a sign $(-)$ for the orientation of movements is shown.

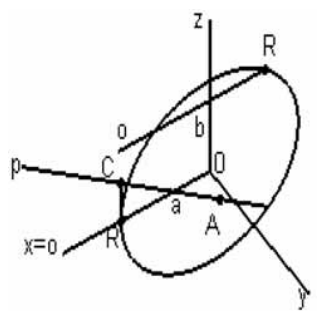

Fig. 13

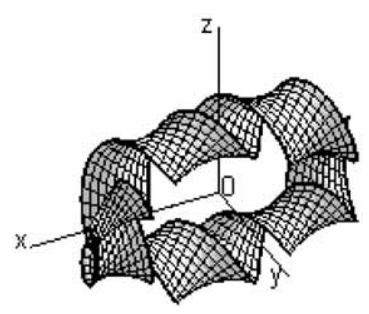

Fig. 14

\subsection{Surfaces of the subgroup III ( $o$ is a normal to the ellipse $k$ )}

We will formulate the transformation matrix of the revolution surface with the axis $o=n$ as a product of matrices which represents the displacement parallel to the vector $(-a, 0,0)$, the revolution through the angle $\epsilon^{\prime}= \pm m \epsilon, \epsilon \in\langle 0,2 \pi\rangle$ about the co-ordinate axis $x$, the displacement parallel to the vector $(d, 0,0)$, where $d=\left|O^{\prime} R\right|, O^{\prime}=n \cap x$, the revolution through the angle $\epsilon^{\prime \prime}$ about the coordinate axis y and displacement parallel to the vector $\left(d^{\prime}, 0,0\right), d^{\prime}=\left|O O^{\prime}\right|$ (Fig. 15)

$$
T(\epsilon)=\left(\begin{array}{cccc}
\cos \epsilon^{\prime \prime} & 0 & \sin \epsilon^{\prime \prime} & 0 \\
\sin \epsilon^{\prime} \sin \epsilon^{\prime \prime} & \cos \epsilon^{\prime} & -\sin \epsilon^{\prime} \cos \epsilon^{\prime \prime} & 0 \\
-\cos \epsilon^{\prime} \sin \epsilon^{\prime \prime} & \sin \epsilon^{\prime} & \cos \epsilon^{\prime} \cos \epsilon^{\prime \prime} & 0 \\
\left.(d-a) \cos \epsilon^{\prime \prime}+d^{\prime}\right) & 0 & (d-a) \sin \epsilon^{\prime \prime} & 1
\end{array}\right)
$$

where $\epsilon^{\prime \prime}= \pm \operatorname{arcos} \frac{b \cos \epsilon}{\sqrt{a^{2} \sin ^{2} \epsilon+b^{2} \cos ^{2} \epsilon}},+$ for $\epsilon \in\langle 0, \pi\rangle$,

- for $\epsilon \in\langle\pi, 2 \pi\rangle, d=\frac{b}{a} \sqrt{a^{2} \sin ^{2} \epsilon+b^{2} \cos ^{2} \epsilon}$,

$d^{\prime}=\frac{a^{2}-b^{2}}{a} \cos \epsilon$

The direction vector of the tangent is $t(-a \sin \epsilon, 0, b \cos \epsilon)$, the direction vector of the normal is $n(-b \cos \epsilon, 0,-a \sin \epsilon)$.

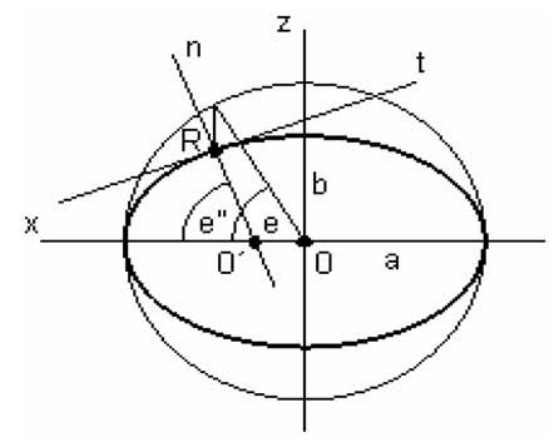

Fig. 15
The straight line $p$ parallel to the axis $o$ (Fig. 16) and given by the point $A\left(a, y_{A}, 0\right)$ creates the surface of the cylindrical type for the sign ( + ) for orientation of motions and for $m=5$ which is shown in Fig. 17.

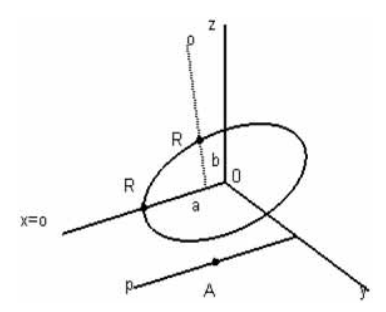

Fig. 16

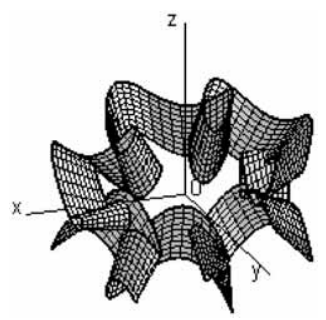

Fig. 17
The straight line $p$ concurrent to the axis $o$ creates the surface of the conical type (Fig. 18). If $p \perp o p \subset x z$ and $x_{A}=a$, the straight line $p$ is a tangent to the ellipse $k$ and it creates the surface of the conical type (Fig. 19).

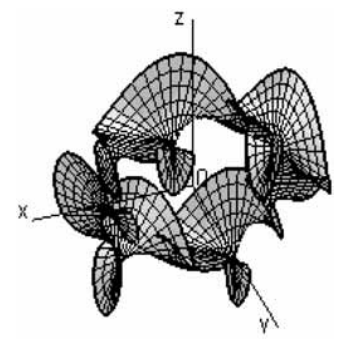

Fig. 18

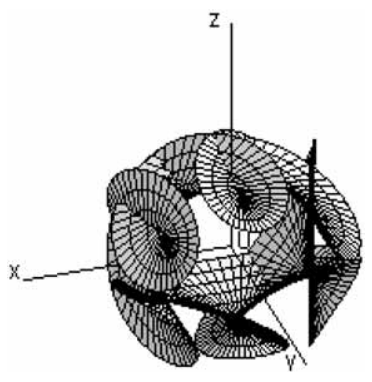

Fig. 19
The surface of the hyperbolical type created by $p / o$ for $m=4$ and equal orientation of the movements is shown in Fig. 21.

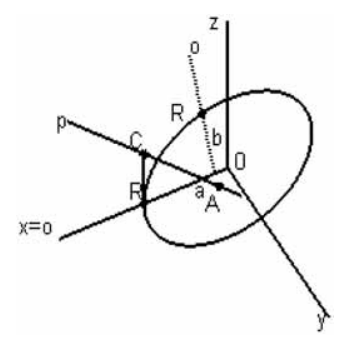

Fig. 20

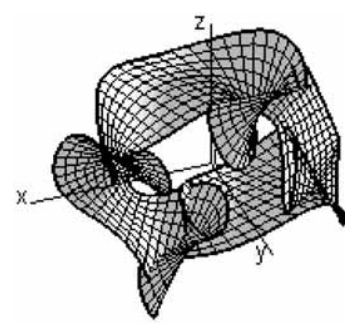

Fig. 21

\subsection{Surfaces of the subgroup IV ( $o$ is a tangent to the ellipse $k$ )}

We will formulate the transformation matrix of the surface created by a revolution for $o=t$ as a product of matrices, which 
represents displacement parallel to the vector $(-a, 0,0)$, the revolution through the angle $\epsilon^{\prime}= \pm m \epsilon, \epsilon \in\langle 0,2 \pi\rangle$ about the coordinate axis $z$, displacement parallel to the vector $(d, 0,0)$, where $d=\left|O^{\prime} R\right|, O^{\prime}=n \cap x$ the revolution through the angle $\epsilon^{\prime \prime}$ about the co-ordinate axis $y$ and displacement parallel to the vector $\left(d^{\prime}, 0,0\right), d^{\prime}=\left|O O^{\prime}\right|($ Fig. 15)

$$
T(\epsilon)=\left(\begin{array}{cccc}
\cos \epsilon^{\prime} \cos \epsilon^{\prime \prime} & \sin \epsilon^{\prime} & \cos \epsilon^{\prime} \sin \epsilon^{\prime \prime} & 0 \\
-\sin \epsilon^{\prime} \cos \epsilon^{\prime \prime} & \cos \epsilon^{\prime} & -\sin \epsilon^{\prime} \sin \epsilon^{\prime \prime} & 0 \\
-\sin \epsilon^{\prime \prime} & 0 & \cos \epsilon^{\prime \prime} & 0 \\
u_{1} & -a \sin \epsilon^{\prime} & u_{2} & 1
\end{array}\right)
$$

where $u_{1}=\left(-a \cos \epsilon^{\prime}+d\right) \cos \epsilon^{\prime \prime}+d^{\prime}, u_{2}=\left(-a \cos \epsilon^{\prime}+d\right) \sin \epsilon^{\prime \prime}$, $\epsilon^{\prime \prime}= \pm \operatorname{arcos} \frac{b \cos \epsilon}{\sqrt{a^{2} \sin ^{2} \epsilon+b^{2} \cos ^{2} \epsilon}},+$ for $\epsilon \in\langle 0, \pi\rangle$,

- for $\epsilon \in\langle\pi, 2 \pi\rangle, d=\frac{b}{a} \sqrt{a^{2} \sin ^{2} \epsilon+b^{2} \cos ^{2} \epsilon}$,

$d^{\prime}=\frac{a^{2}-b^{2}}{a} \cos \epsilon$

The straight line $p \| o$ (Fig. 22) creates the surface of the cylindrical type (Fig. 23). The straight line $p \times o$ creates the surface of the conical type (Fig. 24) and the straight line $p / o$ creates the surface of the hyperbolical type (Fig. 25).

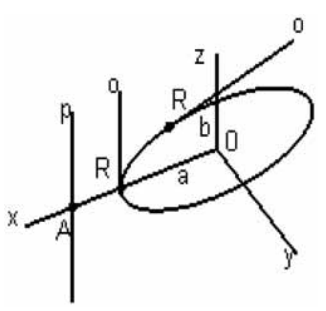

Fig. 22

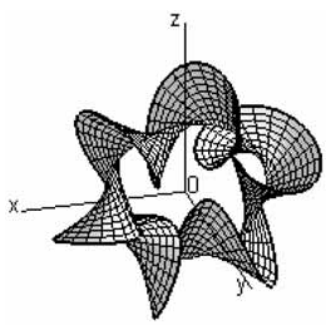

Fig. 24

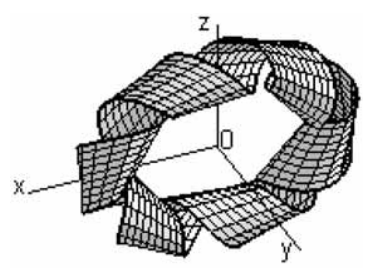

Fig. 23

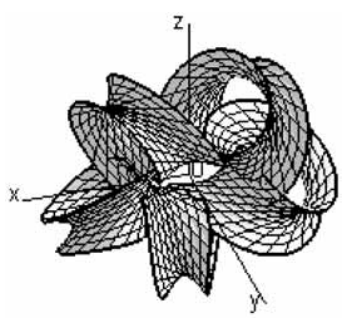

Fig. 25

\subsection{Surfaces of the subgroup $V$ ( $o$ is perpendicular to the plane of ellipse $x z$ )}

We will formulate the transformation matrix of the surface created by a revolution of straightline $p$ around axis $o \perp x z$ as a product of matrices, which represents the displacement parallel to the vector $(-a, 0,0)$, a revolution through the angle $\epsilon^{\prime}= \pm m \epsilon$, $\epsilon \in\langle 0,2 \pi\rangle$, about the coordinate axis $y$, displacement parallel to the vector

$$
T(\epsilon)=\left(\begin{array}{clcl}
\cos \epsilon^{\prime} & 0 & \sin \epsilon^{\prime} & 0 \\
0 & 1 & 0 & 0 \\
-\sin \epsilon^{\prime} & 0 & \cos \epsilon & 0 \\
a\left(\cos \epsilon-\cos \epsilon^{\prime}\right) & 0 & b \sin \epsilon-a \sin \epsilon^{\prime} & 1
\end{array}\right) .
$$

The straight line $p$ parallel to the axis $o$ creates the surface of the cycloidal type and $\epsilon$-curves of this surface are similar to epicycloids or hypocycloids, i.e. the curves which are created by roll a circle along ellipse. The surface in Fig. 26 is of the epicycloidal type, where orientation of the motions is equal and surface in Fig. 27 is of the hypocycloidal type, where orientation of the motions is different.

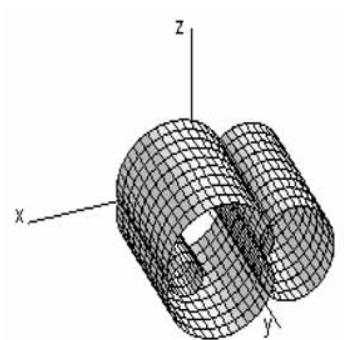

Fig. 26

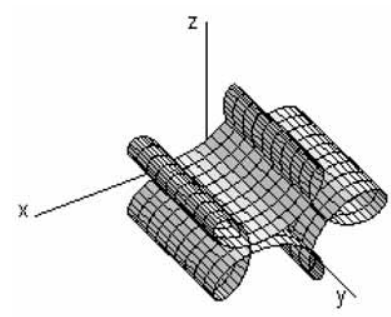

Fig. 27
The surface of the conical type, which is created by the straight line $p$ concurent to the axis $o$ and has the sign $(+)$ for orientation of motions, is the surface of the epicycloidal type (Fig. 28). The surface for the sign $(-)$ is the surface of the hypocycloidal type (Fig. 29).

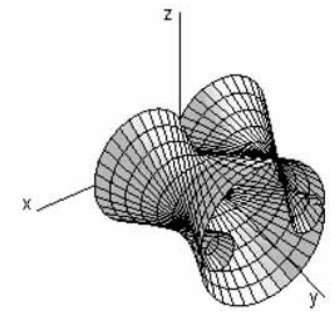

Fig. 28

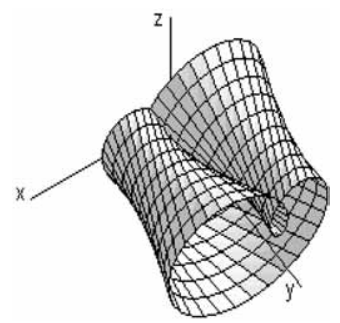

Fig. 30

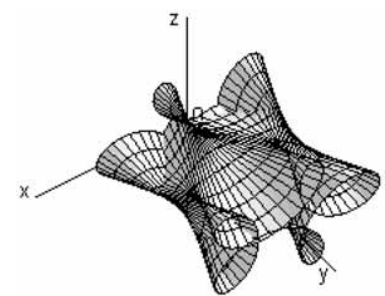

Fig. 29

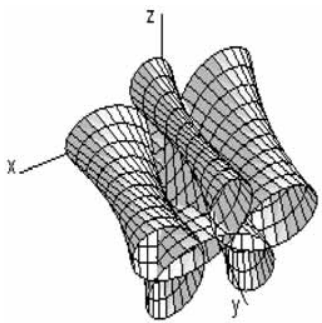

Fig. 31 
The surface of the hyperbolical type, which is created by the straight line $p$ skew to the axis $o$ and has the sign $(+)$ for orientation of motions is the surface of the epicycloidal type (Fig. 30), the surface for the sign $(-)$ is the surface of the hypocycloidal type (Fig. 31).

\section{Conclusion}

We searched the group of linear revolution surfaces which were generated by a straight line revolution with a revolution axis of moving in contact with a curve. We specified the ellipse as a generating curve, which lies in the plane $x z$, its center is congru- ent to the origin $O$ of coordinate system and its major axis is congruent to the axis $x$, the minor axis is congruent to $z$.

The coordinate axis $y$ is perpendicular to the ellipse plane $x z$. If we search relative positions of the axis $o$ and coordinate axis $y$, we can classify these surfaces by three subgroups. The first subgroup contains surfaces of the spherical type with above mentioned concurrent lines, the second subgroup contains surfaces of the Euler type with above mentioned skew lines and the third subgroup contains surfaces of the cycloidal type with above mentioned parallel lines. The surface-type also changes depending upon relative positions of the straight line $p$ and the axis $o$. For better visualization, only particular segments of each surface displayed.

This work was solve by the Grant VEGA 1/4002/07.

\section{References}

[1] GRANÁT, L., SECHOVSKÝ, H.: Computer graphic (in Czech), SNTL Nakladatelství technické literatury, Praha, 1980

[2] DRS, L.: Surface in computer graphic (in Czech), SNTL Nakladatelství technické literatury, Praha, 1984

[3] VELICHOVÁ, D.: Biaxial rotary surfaces (in Slovak), Sborník příspěvku, 24. roč. mezinárodní konference Geometrie a počítačová grafika, Jeseníky, 2004, ISBN 80-248-0581-2

[4] VELICHOVÁ, D.: Biaxial rotary surfaces II (in Slovak), Sborník 25. konference o geometrii a počítačové grafice, ČR, 2005, ISBN 80-7015-013-0

[5] BUDINSKÝ, B., KEPR, B.: Basis of differential geometry with technical applications (in Czech), SNTL Nakladatelství technické literatury, Praha, 1970. 